九州大学学術情報リポジトリ

Kyushu University Institutional Repository

\title{
STOCHASTIC GAME MODELS FOR THE DETERMINATION OF THE OPTIMAL CONTINUOUS SAMPLING INSPECTION PLANS
}

Sakamoto, Takeshi

Kyushu Institute of Design

Kurano, Masami

Department of Mathematics, Faculty of Education, Chiba University

https://doi.org/10.5109/13120

出版情報 : 統計数理研究. 18 (1/2)，pp.9-19，1978-03. Research Association of Statistical Sciences

バージョン：

権利関係 : 


\title{
STOCHASTIC GAME MODELS FOR THE DETERMINATION OF THE OPTIMAL CONTINUOUS SAMPLING INSPECTION PLANS
}

\author{
By \\ Takeshi Sakamoto* \\ and \\ Masami KuRANo** \\ (Received August 10, 1977)
}

\section{Introduction}

In 1943 Dodge [2] published a sampling inspection plan for a continuous production line in which the fraction defective is unknown. Liberman and Solmon [5] and Derman, Littauer and Solmon [1] published the Multi-Level Plan, namely MLP, allowing for any number of sampling levels, and discussed the three types of MLP; MLP- $r \times 1$ Plan, MLP- $r \times s$ Plan and MLP-T Plan, and computed the average outgoing quality, $\mathrm{AOQ}$, and the average outgoing quality limit, $\mathrm{AOQL}$, and tried to obtain the optimal sampling inspection plan. White [7] presented Markovian Decision Models for determining the AOQL of Dodge-type continuous sampling inspection plans.

In this paper, we introduce the inspection cost $c(>0)$, the penalty cost $d(>0)$ and the reward $r(>0)$ which is obtained when finding a defective by the inspection of the item and we give the stochastic game model by which we can determine the optimal continuous inspection plan both in the statistical control and in the nonstatistical control.

\section{Stochastic game model}

In this section, we formulate a stochastic game model, which is investigated in section 3 and used in section 4 for the determination of the optimal continuous inspection plan. $[1,2, \cdots, N]$ is a state space, the set of state of a system and for each state $i, A_{i}$ is the set of actions available to Player 1 (decision maker) at state $i$.

* Kyushu Institute of Design, Fukuoka.

** Department of Mathematics, Faculty of Education, Chiba University, Chiba. 
We set

$$
S_{N}=\left[p=\left(p_{1}, p_{2}, \cdots, p_{N}\right) / \sum_{i=1}^{N} p_{i}=1, p_{i} \geqq 0\right]
$$

For any state $i$ and any action $a \in A_{i}, S_{i}^{a}$ is a subset of $S_{N}$, the set of actions available to Player 2 (state of nature) when the state of a system is $i$ and Player 1 takes the action $a \in A_{i}$. For each state $i, r_{i}$, the reward function at state $i$, is a bounded continuous function on

$$
\bigcup_{a \in A_{i}} \bigcup_{p \in s_{i}^{a}}(a, p) .
$$

The stochastic game is played in a sequence of steps: at each step the play is said to be in some state $i$, Player 1 chooses an action $a$ from $A_{i}$ and then Player 2 chooses an action $p$ from $S_{i}^{a}$ after knowing that Player 1 has chosen the action $a$. As a consequence of the actions chosen by the Players, two things happen: Player 2 pays Player $1 r_{i}(a, p)$ units of money, and the system moves to a new state $j$ according to the distribution $p=\left(p_{1}, p_{2}, \cdots, p_{N}\right)$, the action chosen by Player 2 .

Then, the whole process is repeated from the new state $j$. We henceforth assume that for any state $i$ and any action $a \in A_{i}, A_{i}$ is a finite set and $S_{i}^{a}$ is a compact set. A pure strategy of Player 1 for such a process is defined by a set of functions

$$
\left[F_{t}\right]_{t=0}^{\infty}, F_{t}\left(X_{0}, \Delta_{0}, \Sigma_{0}, \cdots, X_{t}\right) \in A_{X_{t}} \quad \text { for } t=0,1, \cdots
$$

where $X_{t}$ is the state occurring at step $t, \Delta_{t}$ is the action chosen by Player 1 and $\Sigma_{t}$ is the action chosen by Player 2 at step $t$. A strategy $\left[F_{t}\right]_{\ell=0}^{\infty}$ is said to be stationary if there is a map $F$ such that $F(i) \in A_{i}$ for all state $i$ and

$$
F_{t}\left(X_{0}, \Delta_{0}, \Sigma_{0}, \cdots, X_{t}\right)=F\left(X_{t}\right) \in A_{X_{t}} \quad \text { for all } t=0,1,2, \cdots \text {; }
$$

and in this case, $\left[F_{t}\right]$ is denoted by $F$. A pure strategy of Player 2 is defined by a set of functions

$$
\left[F_{t}^{\prime}\right]_{t=0}^{\infty}, F_{t}^{\prime}\left(X_{0}, \Delta_{0}, \Sigma_{0}, \cdots, X_{t}, \Delta_{t}\right) \in S_{X_{t}^{t}}^{\Delta} \quad \text { for } t=0,1,2, \cdots
$$

where $X_{t}, \Delta_{t}$ and $\Sigma_{t}$ are defined as before. A strategy $\left[F_{t}^{\prime}\right]$ is said to be stationary if there is a map $F^{\prime}$ such that $F^{\prime}\left(i, a_{i}\right) \in S_{i}^{a}$ for all state $i$ and all $a_{i} \in A_{i}$ and

$$
F_{t}^{\prime}\left(X_{0}, \Delta_{0}, \Sigma_{0}, \cdots, \Delta_{t}, X_{t}\right)=F^{\prime}\left(X_{t}, \Delta_{t}\right) \in S_{X_{t}^{t}}^{\Delta_{t}} \quad \text { for } t=0,1,2, \cdots ;
$$

and, in this case, $\left[F_{t}^{\prime}\right]$ is denoted by $F^{\prime}$. The set of all behavior strategies of Player 1 is denoted by $C$ and that of Player 2 is denoted by $C^{\prime}$.

A pair $\left(\left[F_{t}\right],\left[F_{t}^{\prime}\right]\right)$ of strategies for Players 1 and 2 associates with each initial state $i$ an $t$-th step expected reward $E_{i}\left(R_{t}\right)$ for Player 1 and the payoff for Player 1 ,

$$
g_{i}\left(\left[F_{t}\right],\left[F_{t}^{\prime}\right]\right)=\liminf _{T \rightarrow \infty} \frac{1}{T+1} \sum_{t=0}^{T} E_{i}\left(R_{t}\right) .
$$

The payoff is therefore the average reward per step. 
If

$$
g_{i}\left(\left[F_{t}\right],\left[\tilde{F}_{t}^{\prime}\right]\right) \leqq g_{i}\left(\left[\tilde{F}_{t}\right],\left[\tilde{F}_{t}^{\prime}\right]\right) \leqq g_{i}\left(\left[\tilde{F}_{t}\right],\left[F_{t}^{\prime}\right]\right)
$$

for all $\left[F_{t}\right] \in C$, all $\left[F_{t}^{\prime}\right] \in C^{\prime}$ and all state $i$, then the strategy $\left[\tilde{F}_{t}\right]$ is optimal for Player 1 and the strategy $\left[\tilde{F}_{t}^{\prime}\right]$ is optimal for Player 2. In this case, we shall say that the stochastic game has a value, $G\left(\left[\tilde{F}_{t}\right],\left[\tilde{F}_{t}^{\prime}\right]\right)$.

\section{The solution of stochastic game}

In this section, we discuss the stochastic game defined in section 2. For two row vectors, $x=\left(x_{1}, x_{2}, \cdots, x_{N}\right), y=\left(y_{1}, y_{2}, \cdots, y_{N}\right)$, the inner product of $x$ and $y$ is denoted by $(x, y) ;(x, y)=\sum_{i=1}^{N} x_{i} y_{i}$. A method due to Howard [4] will be used to prove the following.

LEMMA 1. For each state $i$, there exists some $a_{i} \in A_{i}$ satisfying the conditions that there are two row vectors, $h=\left(h_{1}, h_{2}, \cdots, h_{N}\right), y=\left(y_{1}, y_{2}, \cdots, y_{N}\right)$, such that

$$
\begin{aligned}
& (p, h) \geqq h_{i} \text { for all } p \in S_{i}^{a_{i}} \text { and all } i \text { and, } \\
& r_{i}\left(a_{i}, p\right)+(p, y) \geqq h_{i}+y_{i} \text { for all } i \text { and all } p \in S_{i}^{a_{i}},
\end{aligned}
$$

then, if we define the stationary strategy $F^{*}$ of Player 1 by setting $F^{*}(i)=a_{i}$ for all $i$, it holds that

$$
g_{i}\left(F^{*},\left[F_{t}^{\prime}\right]\right) \geqq h_{i} \text { for all }\left[F_{t}^{\prime}\right] \in C^{\prime} \text { and all } i \text {. }
$$

Proof. Let $\left[F_{t}^{\prime}\right]$ be any strategy in $C^{\prime}$ and let $p_{t}^{*}$ denote the $t$-th step action chosen by $\left[F_{t}^{\prime}\right]$. The expectation $E(\bullet)$ is made for the stochastic process obtained by the use of the pair $\left(F^{*},\left[F_{t}^{\prime}\right]\right)$.

We prove the following assertion at first.

$$
E\left(h_{X_{t}} / X_{0}=i\right) \geqq h_{i} \text { for all state } i \text {. }
$$

We will prove (A) by induction. Assume that (A) is true for $t=t-1$. From (1) and the assumption of induction,

$$
\begin{aligned}
E\left(h_{X_{t}} / X_{0}=i\right) & =E\left(E\left(\left(p_{t-1}^{*}, h\right) / X_{t-1}, X_{0}=i\right)\right) \\
& \geqq E\left(h_{X_{t-1}} / X_{0}=i\right) \\
& \geqq h_{i} .
\end{aligned}
$$

From (2), we have, at the $t$-th step,

$$
r_{X_{t}}\left(F^{*}\left(X_{t}\right), p_{t}^{*}\right)+\left(p_{t}^{*}, y\right) \geqq h_{X_{t}}+y_{X_{t}} .
$$

By taking the expectation and summing up,

$$
\begin{gathered}
\sum_{t=0}^{T} E\left(r_{X_{t}}\left(F^{*}\left(X_{t}\right), p_{t}^{*}\right) / X_{0}=i\right)+\sum_{t=0}^{T} E\left(\left(p_{t}^{*}, y\right) / X_{0}=i\right) \\
\geqq \sum_{i=0}^{T} E\left(h_{X_{t}} / X_{0}=i\right)+\sum_{t=0}^{T} E\left(y_{X_{t}} / X_{0}=i\right) .
\end{gathered}
$$

Since $E\left(\left(p_{t+1}^{*}, y\right) / X_{0}=i\right)=E\left(y_{X_{t+1}} / X_{0}=i\right)$, 
we get, from (A),

$$
\sum_{t=0}^{r} E\left(r_{X_{t}}\left(F^{*}\left(X_{t}\right), p_{t}^{*}\right) / X_{0}=i\right)+E\left(y_{X_{T+1}} / X_{0}=i\right) \geqq(T+1) h_{i}+y_{i} .
$$

Therefore,

$$
\lim _{T \rightarrow \infty} \inf \frac{1}{T+1} \sum_{t=0}^{T} E\left(r_{X_{t}}\left(F^{*}\left(X_{t}\right), p_{t}^{*}\right) / X_{0}=i\right) \geqq h_{i} \quad \text { for all state } i \quad \text { Q.E. D. }
$$

We shall investigate the following mathematical programming problem in order to solve the stochastic game.

PROBLEM 1:

Maximize $\min \left[h_{1}, h_{2}, \cdots, h_{N}\right]$

subject to the conditions of Lemma 1 .

By setting $H=\min \left[h_{1}, h_{2}, \cdots, h_{N}\right]$, it is easy to show that Problem 1 is equivalent to the following Problem 2.

PROBLEM 2:

Maximize $H$

subject to that, for each state $i$, there exists some $a_{i} \in A_{i}$

satisfying the conditions that there is a row vector $y=\left(y_{1}, y_{2}, \cdots, y_{N}\right)$ such that

$$
r_{i}\left(a_{i}, p\right)+(p, y) \geqq H+y_{i} \text { for all } i \text { and all } p \in S_{i}^{a} i .
$$

We henceforth assume the following irreducibility property: For any stationary strategy pair $\left(F, F^{\prime}\right)$ of Players 1 and 2, stochastic matrix $P\left(F, F^{\prime}\right)=\left[\begin{array}{c}p_{1} \\ \vdots \\ p_{N}\end{array}\right] \quad$ is irreducible; ie for any $i$ and $j$, the probability that state $j$ will be reached from the initial state $i$ in some $n$ steps is positive, where $p_{i}=F^{\prime}(i, F(i))$ for all $i$. On this assumption, for any stationary $F, F^{\prime}$, the payoff $g_{i}\left(F, F^{\prime}\right)$ is independent of $i$ and is given by

$$
\sum_{j=1}^{N} \pi_{j} r_{j}\left(F(j), F^{\prime}(j, F(j))\right)
$$

where $\left(\pi_{1}, \pi_{2}, \cdots, \pi_{N}\right)$ is the unique positive vector of stationary probabilities. Assuming the irreducibility property, we can put Lemma 1 in the following Lemma 2.

LEMMA 2. If there exists a stationary strategy $F$ of Player 1 satisfying the conditions that there are a constant $H$ and a row vector $y=\left(y_{1}, y_{2}, \cdots, y_{N}\right)$ such that

$$
\min _{F \in S_{i}^{F(i)}}\left[r_{i}(F(i), p)+(p, y)\right] \geqq H+y_{i} \text { for all } i \text {, }
$$

then,

$H \leqq \inf _{\left[F_{t}^{\prime}\right] \in C^{\prime}} G\left(F,\left[F_{t}^{\prime}\right]\right)$ with strict inequality if (4) is with strict inequality for some $i$, where $G\left(F,\left[F_{t}^{\prime}\right]\right)=\min _{i} g_{i}\left(F,\left[F_{t}^{\prime}\right]\right)$. 
The inequality (3) in Problem 2 is changed to equality by proving the following Lemma 3.

LEMma 3. If the conditions of Lemma 2 is satisfied, there exist a constant $\vec{H}$ and a row vector $\bar{y}=\left(\bar{y}_{1}, \bar{y}_{2}, \cdots, \bar{y}_{N}\right)$ such that

(5) $\min _{P \in S_{i}^{F(i)}}\left[r_{i}(F(i), p)+(p, \bar{y})\right]=\bar{H}+\bar{y}_{i}$ for all $i$ and,

(6) $\bar{H} \geqq H$ with strict inequality if (4) is with strict inequality for some $i$.

ProOF. We set

$$
\begin{aligned}
& \inf _{F^{\prime} \in C^{\prime}} G\left(F, F^{\prime}\right)=G\left(F, \tilde{F}^{\prime}\right)=\bar{H} . \\
& \tilde{F}^{\prime}: \text { stationary }
\end{aligned}
$$

Then, from Lemma 2, we have $H \leqq \bar{H}$, where the strict inequality holds when (4) is with strict inequality for some $i$.

Setting $p_{i}^{*}=\tilde{F}^{\prime}(i, F(i)) \in S_{i}^{F^{(i)}}$, by Howard [6], there exists a vector $\bar{y}=\left(\bar{y}_{1}, \bar{y}_{2}, \cdots\right.$, $\left.\bar{y}_{N}\right)$ such that

$$
r_{i}\left(F(i), p_{i}^{*}\right)+\left(p_{i}^{*}, \bar{y}\right)=\bar{H}+\bar{y}_{i} \text { for all } i .
$$

If, for each state $i$, there exists $p_{i} \in S_{i}^{F^{(i)}}$ such that

$$
r_{i}\left(F(i), p_{i}\right)+\left(p_{i}, \bar{y}\right) \leqq \bar{H}+\bar{y}_{i} \text { for all } i
$$

and (7) is with strict inequality for some $i$, by the same discussion as Lemma 2, we get

$G\left(F, F^{\prime \prime}\right)<\bar{H}$, where $F^{\prime \prime}$ is any stationary strategy satisfying $F^{\prime \prime}(i, F(i))=p_{i}$. This is the contradiction. Therefore,

$$
\min _{p \in S_{i}^{(i)}}\left[r_{i}(F(i), p)+(p, \bar{y})\right]=\bar{H}+\bar{y}_{i} \text { for all } i \text {. Q.E.D. }
$$

The following Lemma is a version of Theorem 4 of Hoffman and Karp [3] and necessary to prove the theorem.

LEMMA 4. If both $(H, y)$ and $(\bar{H}, \bar{y})$ satisfy the following equations

$$
\max _{a_{i} \in A_{i}} \min _{P \in S_{i}^{a} i}\left[r_{i}\left(a_{i}, p\right)+(p, y)\right]=H+y_{i} \text { for all } i,
$$

there, $H=\bar{H}$ and there is a constant $C$ such that $y_{i}-\bar{y}_{i}=C$ for all $i$.

ProOF. For each $i$, choose $a_{i}^{*}$ and $p_{i}^{*}$ such that

and

$$
\max _{a_{i} \in \boldsymbol{A}_{i}} \min _{P \in S_{i}^{a_{i}}}\left[r_{i}\left(a_{i}, p\right)+(p, y)\right]=\min _{\boldsymbol{P} \in S_{i}^{a_{i}}}\left[r_{i}\left(a_{i}^{*}, p\right)+(p, y)\right]
$$

$$
\min _{P \in S_{i}^{a_{i}}}\left[r_{i}\left(a_{i}^{*}, p\right)+(p, \bar{y})\right]=r_{i}\left(a_{i}^{*}, p_{i}^{*}\right)+\left(p_{i}^{*}, \bar{y}\right) .
$$

Then, we have

$$
\begin{aligned}
& r_{i}\left(a_{i}^{*}, p_{i}^{*}\right)+\left(p_{i}^{*}, y\right) \geqq H+y_{i} \\
& r_{i}\left(a_{i}^{*}, p_{i}^{*}\right)+\left(p_{i}^{*}, \bar{y}\right) \leqq \bar{H}+\bar{y}_{i} .
\end{aligned}
$$


Therefore,

$$
\bar{H}-H+\left(\bar{y}_{i}-y_{i}\right) \geqq\left(p_{i}^{*}, \bar{y}-y\right) \text { for all } i \text {. }
$$

If $\bar{H}-H$ is negative, $\left(\bar{y}_{i}-y_{i}\right)>\left(p_{i}^{*}, \bar{y}-y\right)$ for all $i$. This means that each component of the vector

$$
\bar{y}-y=\left(\bar{y}_{1}-y_{1}, \cdots, \bar{y}_{N}-y_{N}\right)
$$

exceeds a convex combination of components. Since this is impossible, $\bar{H}-H \geqq 0$.

We can establish similarly that $\bar{H}-H \leqq 0$. Therefore, $\bar{H}=H$, and setting $\bar{H}-H$ equal to zero, we have

$$
\bar{y}-y \geqq P(\bar{y}-y),
$$

where

$$
P=\left[\begin{array}{c}
p_{1}^{*} \\
\vdots \\
p_{N}^{*}
\end{array}\right] .
$$

By the irreducibility assumption, $P$ is an irreducible Markov matrix, so that the only solution to (9) are those in which all component of $\bar{y}-y$ are equal. Q.E.D.

The above lemma will now be used to prove Theorem.

THEOREM: Let the irreducibility assumption be satisfied.

(a) $(H, y)$ is an optimal solution of Problem 2 if and only if $(H, y)$ is a solution of the equations (8),

(b) and then, $H$ is a game value and a stationary strategy pair $\left(\tilde{F}, \tilde{F}^{\prime}\right)$ attaining the left side of (8) is an optimal strategy pair of Players 1 and 2.

Proof. For (a), let $(H, y)$ be an optimal solution of Problem 2. If, for each $i$, there exists $a_{i} \in A_{i}$ such that

$$
\min _{\boldsymbol{F} \in S_{i}^{a}{ }_{i}}\left[r_{i}\left(a_{i}, p\right)+(p, y)\right] \geqq H+y_{i} \text { for all } i
$$

and with strict inequality for some $i$, from Lemma 3 , there exist a constant $\bar{H}$ and a row vector $\bar{y}$ such that $\bar{H}>H$ and

$$
\min _{P \in s_{i}^{a_{i}}}\left[r_{i}\left(a_{i}, p\right)+(p, y)\right]=\bar{H}+\bar{y}_{i} \text { for all } i .
$$

This is the contradiction. The converse is obvious from Lemma 4.

For (b), setting $p\left(i, a_{i}\right)=\tilde{F}^{\prime}\left(i, a_{i}\right)$, from the definition,

$$
r_{i}\left(a_{i}, p\left(i, a_{i}\right)\right)+\left(p\left(i, a_{i}\right), y\right) \leqq H+y_{i} \text { for all } a_{i} \in A_{i} \text { and all } i .
$$

By the same discussion as Lemma 1, we can show that

$$
g_{i}\left(\left[F_{t}\right], \tilde{F}^{\prime}\right) \leqq H \text { for all }\left[F_{t}\right] \in C .
$$

On the other hand, we can show by the similar way that

$$
g_{i}\left(\tilde{F},\left[F_{t}^{\prime}\right]\right) \geqq H \text { for all }\left[F_{t}^{\prime}\right] \in C^{\prime} \text { and all } i .
$$

Thus, $H$ is a game value and $\left(\widetilde{F}, \widetilde{F}^{\prime}\right)$ is an optimal pair of Player 1 and 2. Q. E.D. 


\section{Optimal continuous sampling inspection plan $(\mathrm{n}, \mathrm{f}, \mathrm{N})$}

In this section, we consider an optimal continuous sampling inspection plan. We assume the items are classified, after measurement, as "defective" or "non-defective". The two most common methods of partial inspection are block sampling and probability sampling.

Block sampling at rate $A$ is that one item is chosen at random for inspection from consecutive blocks of length $A$, and Probability sampling at rate $A$ is that successive items are inspected with probability $A$. For any given $(n, f, N)$, where $n$ and $N$ are positive integers and $0<f<1$, the levels are as follows:

level (0) Inspect $100 \%$ of the out-put

level (1) Inspect by block sampling at rate $\left(\frac{1}{f}\right)$ or by probability sampling at rate $f$

level $(k) \quad$ Inspect by block sampling at rate $\left(\frac{1}{\bar{f}}\right)^{k}$ or by probability sampling at rate $(f)^{k}$

level $(N) \quad$ Inspect by block sampling at rate $\left(\frac{1}{\bar{f}}\right)^{N}$ or by probability sampling at rate $(f)^{N}$

The MLP $-r \times 1$ Plan published by Derman, Littauer and Solmon [1] is as follows: We say we are in level $(j)$. If $n$ successively inspected items are found clear of defects, begin sampling at the level $(j+1)$. On the other hand, if a defective item is found before this is accomplished, revert immediately to the level $(j-r)$, if $j>r$, or to the level $(0)$, this is, one hundred percent inspection if $j \leqq r$. If a non-defective is found in level $(N)$, we remain in level $(N)$. Let us obtain an optimal sampling inspection plan. We set the state space $=\left[E_{00}, E_{01}, \cdots, E_{0, n-1}, E_{1,0}, \cdots, E_{N 0}\right]$ where $E_{l k}$ is the state that $k$ non-defectives have been observed successively in the level $(l)$.

$A_{E_{l m}}$, the set of actions available to Player 1 (decision maker] in state $E_{l m}$, is

$$
\left[a_{l^{\prime} m^{\prime}}, 0 \leqq l^{\prime}<l, 0 \leqq m^{\prime} \leqq n-1 \text { or } l^{\prime}=l, 0 \leqq m^{\prime} \leqq m\right],
$$

where $a_{l^{\prime} m^{\prime}}$ is the action that we immediately revert to state $E_{l^{\prime} m^{\prime}}$ when a defective is found and go to state $E_{l, m+1}$ when a non-defective is found.

The inspection cost is $c(c>0)$, the penalty cost $d(d>0)$, and the reward is $r(r>0)$ which is obtained when finding a defective by the inspection of the item.

(I) Uncontrolable CASE. If the manifacturing process is not statistically controlled so that the probability of producing a defective unit may vary from step to step, $S_{E_{l m}^{\prime} m^{\prime}}^{a}$, the set of actions available to Player 2 (state of nature) when Player 1 choose the action $a_{l^{\prime} m^{\prime}} \in A_{E_{l m}}$, is 


$$
\begin{aligned}
& {\left[\begin{array}{c}
E_{l^{\prime} m^{\prime}} \\
\vee
\end{array} \stackrel{E_{l, m+1}}{\bigvee}\right.} \\
& \begin{array}{c}
E_{l^{\prime} m^{\prime}} \\
S_{E_{N 0}}^{a_{l^{\prime} m^{\prime}}}=[p=(0, \cdots, 0, p, 0, \cdots, 0,1-p) / 0 \leqq p \leqq 1],
\end{array}
\end{aligned}
$$

where $E_{l n}=E_{l+1,0}$. If, for any $k$, the partial observation in level $(k)$ is block sampling at rate $\left(\frac{1}{f}\right)^{k}$, the reward function is

$$
r_{E_{l m}}\left(a_{l^{\prime} m^{\prime}}, p\right)=p\left(f^{l}-1\right) d+p \cdot f^{l} \cdot r-(1-p) c f^{l} .
$$

If the partial observation in level $(k)$ is probability sampling at rate $(f)^{k}$, the reward function is

$$
r_{E_{l m}}\left(a_{l^{\prime} m^{\prime}}, p\right)=f^{l}[p \cdot r-(1-p) \cdot c]+\left(1-f^{l}\right) p d .
$$

We shall consider the optimal inspection plan in the case of block sampling. The methods in the case of probability sampling is similar to those in the case of block sampling. By Theorem in section 3, it is sufficient to solve the equation (8) to obtain the optimal inspection plan. Since the reward function is linear of $p$, the equation (8) is the following:

$$
\begin{gathered}
\left.\min \left[y_{l, m+1}-c f^{l}, \underset{\substack{0 \leq l^{\prime} \leq l \\
0 \leq m^{\prime} \leq n-1}}{\left.\left[\max _{l^{\prime} m^{\prime}}\right)\right] \vee\left[\max _{0 \leq m^{\prime} \leq m}\right.} y_{l m^{\prime}}\right]+\lambda(l)\right]=H+y_{l m} \\
\text { for } l=0, \cdots, N-1 \quad m=0, \cdots, n-1 \\
\min \left[y_{N 0}-c f^{N}, \max _{l^{\prime}, m^{\prime}}\left(y_{l^{\prime} m^{\prime}}\right)+\lambda(N)\right]=H+y_{N 0}
\end{gathered}
$$

where $a \vee b=\max [a, b]$ and $\lambda(l)=\left(f^{l}-1\right) d+r f^{l}$. It is easy to prove the following two propositions.

Proposition 1. In equation (12), if

$$
y_{00}=0, y_{l, m+1}-c f^{\imath}=H+y_{l m}
$$

for $\quad l<L, m=0, \cdots, n-1$ and for $l=L, m=0$,

then

$$
y_{L, m+1}-c f^{L}=H+y_{L, m} \text { for } \quad m=1,2, \cdots, n-1 .
$$

Proposition 2. For any $l$ and $m$,

$$
\left.\max _{\substack{0 \leq l^{\prime} \leq l-1 \\ 0 \leq m^{\prime} \leq n-1}}\left(y_{l^{\prime} m^{\prime}}\right)\right] \vee\left[\max _{0 \leqq m^{\prime} \leqq m} y_{l m^{\prime}}\right]=\left[\max _{0 \leq l^{\prime} \leqq l}\left(y_{l^{\prime} 0}\right)\right] \vee y_{l m} .
$$

From the above propositions, the solution of (12), setting $y_{00}=0$, is the follows: We set $\nu(l, l-k)=c\left(f^{k}+\cdots+f^{l-1}\right)$, and set

$$
g_{l, k}(c, r, d)=\frac{\lambda(l)-n \nu(l, l-k)}{(l-k) n+1},
$$


if

$$
\begin{gathered}
-[(l-k) n+1] \cdot c \cdot f^{k-1}+n \cdot c \cdot \nu(l, l-k) \leqq \lambda(l) \\
\leqq-[(l-k) n+1] \cdot c \cdot f^{k}+n \cdot c \cdot \nu(l, l-k)
\end{gathered}
$$

$=+\infty$ otherwise

for

$$
k=1, \cdots, l-1, \quad l=1, \cdots, N-1 .
$$

Set

for

$$
\begin{aligned}
& g_{l, 0}(c, r, d)=\frac{\lambda(l)-n \cdot \nu(l, l)}{l \mathrm{n}+1}, \\
& \text { if } \quad \lambda(l) \leqq-[l \cdot n+1] \cdot c+n \nu(l, l) \\
& =+\infty, \quad \text { otherwise }
\end{aligned}
$$

and set

$$
l=0,1, \cdots, N \text {, }
$$

$$
\begin{aligned}
& g_{l, l}(c, r, d)=\lambda(l) \text {, } \\
& \text { if } \\
& \lambda(l)>-c \cdot f^{l-1} \\
& =+\infty \text {, otherwise }
\end{aligned}
$$

for

$$
l=0,1, \cdots, N-1 \text {, }
$$

and

$$
\begin{aligned}
& g_{N}^{\prime}=-c f^{N} \text {, } \\
& \text { if } \\
& \lambda(N)>-c \cdot f^{N}
\end{aligned}
$$

$=+\infty$, otherwise,

We set

$$
\begin{aligned}
G_{l}(c, r, d) & =\max _{0 \leq k \leq l}\left[g_{l, k}(c, r, d)\right] \\
& =g_{l, k_{l}^{*}}(c, r, d) .
\end{aligned}
$$

Then, the value of the stochastic game is

$$
G(c, r, d)=\min _{0 \leq l \leq N}\left[G_{l}(c, r, d)\right] \wedge g_{N}^{\prime}
$$

where

$$
a \wedge b=\min [a, b] .
$$

And the optimal stationary strategy $F^{*}$ of Player 1 is the following

\begin{tabular}{|c|c|}
\hline state & $\cdots E_{l 0}, \cdots E_{l m}, \cdots E_{l+1,0}, \cdots$ \\
\hline$F^{*}$ & $\cdots a_{k^{*} 0}, \cdots a_{k_{l} m}, \cdots a_{k_{l+1}^{*}, 0}, \cdots$ \\
\hline
\end{tabular}


where

$$
a_{k_{l} m}=\left\{\begin{array}{lll}
a_{k_{l} m} & \text { if } & G(c, r, d)>-c f^{l} \\
a_{k_{l}, 0}^{*} & \text { if } & G(c, r, d) \leqq-c f^{1}
\end{array}\right.
$$

Numerical Example. $n=2, \quad f=0.5, \quad N=6$.

Example 1. $\quad d=2, \quad r=1.5, \quad c=1$.

Optimal strategy of Player 1 is given by the following transition diagram.

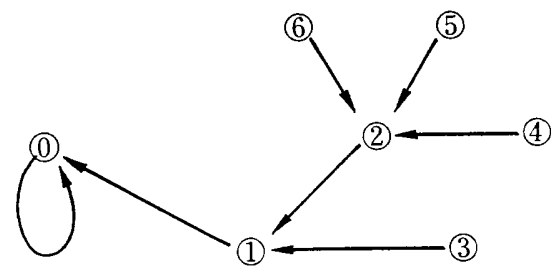

In this diagram (i) $\rightarrow$ (i) shows we revert immediately to the level (j) when a defective is found in the level (i), and value of game is -0.708

Example 2. $\quad d=10, \quad r=1.5, \quad c=1$.

Optimal strategy of Player 1 is given by the following transition diagram.

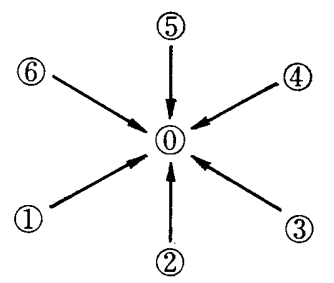

In this example, the MLP-T plan is optimal and value of game is -2.083 .

Example 3. $\quad d=1.5, \quad r=15, \quad c=1$.

Optimal strategy of Player 1 is given by the following diagram.

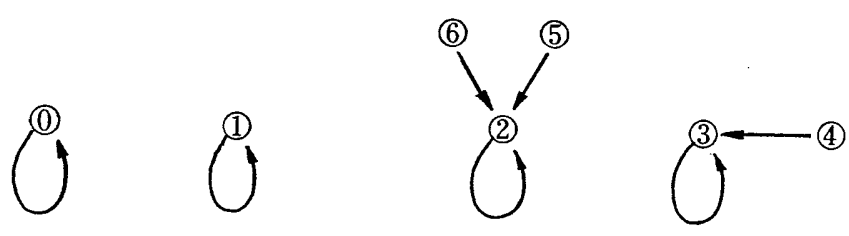

The value of game is -0.279 .

(II) Controlable Case.

If the manufacturing process is statistically controlled so that the probability of producing a defective unit is constant and equal to $p$, where the true value of $p$ is unknown, we set 


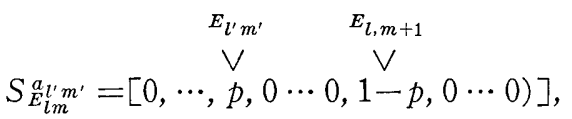

the set consisting of one element. We can get the game value, $G(c, r, d)$, in a same way as the uncontrolable case. Since $G(c, r, d)$ is a function of $p$, we write $G(c, r, d)$ $=G(c, r, d, p)$.

Let

$$
G\left(c, r, d, p^{*}\right)=\min _{0 \leq p \leq 1} G(c, r, d, p) .
$$

Then, $G\left(c, r, d, p^{*}\right)$ is the stochastic game value in the controlable case and optimal stationary strategy of Player 1 is obtained in the same way as the uncontrolable case by setting $p=p^{*}$ in (13)

\section{References}

[1] Derman, C., Littauer, S. and Solomon, H., Tightened-Multi-level continuous sampling plans. Ann. Math. Statist. 28 (1957), 395-404.

[2] Dodge, H.F., A sampling Inspection plan for continuous production. Ann. Math. Statist. 14 (1943), 264-279.

[3] Hoffman, A.J. and KARP, R. M., On non-terminating stochastic games. Magi. Sci. 12, no 5 (1966).

[4] Howard, R., Dynamic Programming and Markov Processes. Wiley, New York, (1960).

[5] Liberman, G. J. and Solomon, H., Multi-level continuous sampling plans. Ann. Math. Statist. 26 (1955), 686-704.

[6] Satia, J.K. and Love, R.E., Markovian Decision Processes with uncertain transition probabilities. Oper. Research. 21 (1973), 728-740.

[7] White, L.S., Markovian Decision Models for evaluation of a large doss of continuous sampling inspection plans. Ann. Math. Statist. (1966), 1408-1420. 